\title{
PREVALÊNCIAS DE SOBREPESO, OBESIDADE E HÁBITOS DE VIDA ASSOCIADOS AO RISCO CARDIOVASCULAR EM ALUNOS DO ENSINO FUNDAMENTAL
}

\author{
Moacyr Roberto Cuce Nobre*, Rachel Zanetta de lima Domingues, Atalanta Ruiz da Silva, Fernando Antonio Basile Colugnati, José Augusto de \\ Aguiar Carrazedo Taddei \\ Trabalho realizado na Unidade de Epidemiologia Clínica do Instituto do Coração - Hospital das Clínicas da Faculdade de Medicina da Universida-
} de de São Paulo.

*Correspondência:

R.Cd Cagliori, 115

Cep: 05454030,

São Paulo, SP

mrcnobre@usp.br

\begin{abstract}
RESUMO
OBjetivo. Estudar a prevalência do risco cardiovascular associado ao estilo de vida de escolares da $5^{a}$ à 8 a série do ensino fundamental público e privado.

Métodos. Foram visitadas 87 salas, selecionadas aleatoriamente, totalizando 2125 estudantes que responderam ao questionário e tiveram o índice de massa corpórea calculado. A amostra representa cerca de $2 \%$ dos alunos de duas regiões de ensino do município de São Paulo.

Resultados. Foram observados 24\% com sobrepeso ou obesidade, 53,3\% com hábito alimentar inadequado, 15,4\% com

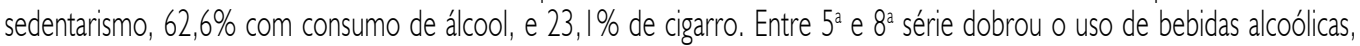
triplicou a proporção de adolescentes do sexo masculino que experimentou o cigarro, e quintuplicou do sexo feminino. Em contraposição, decresceu o hábito alimentar inadequado, embora 40\% dos alunos das escolas públicas, e 58\% das privadas, apresentem essa condição na $8^{a}$ série. $O$ percentual de alunos com sobrepeso e obesidade foi maior nas escolas privadas, com o crescer das séries houve decréscimo dessa proporção, apesar de não atingir nível de significância estatística. 0 sedentarismo foi maior na escola pública e, com o crescer da idade, se mostrou controverso entre a escola pública e privada, aumentando com a idade na pública. As adolescentes do sexo feminino freqüentam menos as aulas de educação física. Hábito alimentar inadequado foi caracterizado por colocar mais sal na comida já preparada, menor consumo de laticínios, frutas e por outro lado, maior consumo de alimentos representados pelos refrigerantes, manteiga e salgadinhos.

Conclusão. O reconhecimento precoce do risco cardiovascular associado ao estilo de vida fundamenta práticas preventivas de educação em saúde nas escolas.

Unitermos: Epidemiologia. Doença cardiovascular. Estilo de vida. Adolescente. Ensino fundamental. Educação em saúde.
\end{abstract}

\section{INTRODUÇÃO}

O conjunto das doenças cardiovasculares representadas pela hipertensão arterial, aterosclerose coronária, doença cerebrovascular e suas complicações constitui a maior causa de morte precoce na idade adulta. De acordo com o Estudo de Framingham, entre as pessoas com 40 anos de idade, sem queixas cardiovasculares, metade dos homens e um terço das mulheres apresentam alguma manifestação da doença da artéria coronária, como angina de peito, insuficiência coronária, infarto do miocárdio ou morte pela doença'.

A cardiopatia isquêmica, com 7,375 milhões de mortes, e a doença cerebrovascular, com 5, 106 milhões, são as principais causas de morte na população mundial no ano de 1998. Segundo a Organização Mundial da Saúde, as duas juntas correspondem a 23\% da mortalidade geral2. Dados demográficos do Estado de São Paulo referentes ao ano de 1998 mostram que as doenças do aparelho circulatório constituem a primeira causa de morte condensada, sendo que $63 \%$ destas correspondem à doença isquêmica do coração e cerebrovascular. Somadas superam em mais de $35 \%$ as neoplasias e causas externas, respectivamente, segunda e terceira causas de morte no Estado ${ }^{3}$. No município de São Paulo, os óbitos por grandes grupos de causas, referentes ao ano de
1997, mostram a mesma ordem de freqüência observada no Estado, sendo as doenças do aparelho circulatório responsáveis por 32,8\% dos 64.034 óbitos, seguidas das neoplasias (16,3\%) e das causas externas $(13,5 \%)^{4}$.

A aterosclerose surge em decorrência da formação de placas de gordura na parede arterial. As lesões avançadas podem obstruir a luz do vaso, resultando em síndromes isquêmicas agudas, que compreendem os quadros de doença arterial coronariana, doença cerebrovascular e doença vascular periférica. Existem evidências anatomopatológicas de que a formação da placa aterosclerótica inicia-se na infância e progride lentamente até a vida adulta ${ }^{5}$.

O Bogalusa Heart Study correlacionou o achado de aterosclerose em necrópsia de crianças com fatores de risco detectados antes da morte, como os níveis séricos elevados de colesterol total, da fração $L D L$, e baixos da fração $H D L^{6}$. O estudo concluiu que fatores de risco estão relacionados com o desenvolvimento de lesões ateroscleróticas, desde os seus estágios mais precoces na infância. Os autores recomendam o rastreamento dos fatores de risco cardiovascular para todas as crianças durante o exame pré-escolar ou mesmo antes.

Com a finalidade de servir como referência para sistemas de vigilância, a Organização Mundial da Saúde classifica os fatores de risco 
que se associam à etiologia das doenças não-transmissíveis em dois grupos, um vinculado ao indivíduo e outro à comunidade ${ }^{7}$. Os fatores de risco estão agrupados como geral, associados ao estilo de vida, e intermediários ou biológicos. No primeiro agrupamento, encontram-se a idade, sexo, escolaridade e herança genética. No segundo, o tabagismo, dieta inadequada, e sedentarismo. No terceiro, a hipertensão arterial, obesidade e hipercolesterolemia. Entre os fatores de risco associados à comunidade estão as condições socioeconômicas, culturais, ambientais e de urbanização.

A aterosclerose coronária e cerebrovascular apresenta associações com fatores de risco bem definidos ${ }^{8}$. Muitos destes fatores de risco são decorrentes do estilo de vida, como padrão alimentar individual ou de grupos populacionais ${ }^{9}$, ingestão de alimentos salgados ${ }^{10}$, tabagismo ${ }^{\prime \prime} \mathrm{e}$ atividade física'2. Meta-análise de estudos observacionais concluiu que os indivíduos sedentários apresentam o dobro do risco para desenvolver evento coronariano comparados aos fisicamente ativos ${ }^{13}$. O consumo elevado de bebidas alcoólicas confere risco para o desenvolvimento de hipertensão arterial, acidente vascular hemorrágico ${ }^{14} \mathrm{e}$ cardiomiopatia ${ }^{15}$.

Nas regiões em desenvolvimento, à medida que suas economias se industrializam, as doenças crônico-degenerativas tornam-se mais prevalentes, principalmente em função da adoção de estilos de vida ocidentalizados, caracterizados por maior proporção de sedentarismo, acompanhados de dietas com mais gordura e menos fibras ${ }^{16}$. Tem sido observado aumento do índice de massa corporal em adolescentes de zona urbana de regiões desenvolvidas do sul e sudeste brasileiro, particularmente do sexo feminino ${ }^{17}$. Estas observações foram posteriormente confirmadas, embora o percentual de sobrepeso e obesidade ocorra em menor magnitude se comparado ao dos países desenvolvidos ${ }^{18}$.

Dois ensaios preventivos randomizados envolvendo milhares de participantes indicam benefícios propiciados por programas que objetivam a mudança de estilo de vida de crianças e adolescentes. $\mathrm{O}$ programa $\mathrm{CATCH}$, desenvolvido em escolas norte-americanas, observou mudanças significantes nos hábitos alimentares e de atividade física, embora não tivesse sido suficiente para reversão dos fatores de risco após dois anos e meio de ensaio ${ }^{19}$. Estudo australiano com pré-adolescentes entre 10 e 12 anos de idade submetidos à intervenção sobre o estilo de vida, que incluiu a prática de atividade física e a orientação alimentar no conjunto familiar, mostrou que os pré-adolescentes considerados de maior risco, devido à condição física, gordura corporal, pressão arterial e dosagem de colesterol, mostraram melhores resultados no controle de fatores de risco e hábitos relacionados à saúde ${ }^{20}$.

Durante a adolescência, são incorporados muitos dos hábitos relacionados à alimentação, prática de atividade física, consumo de cigarros e bebidas alcoólicas, que propiciam o aparecimento ou agravam fatores biológicos de risco cardiovascular passíveis de reversão, como hipertensão arterial, obesidade, hiperinsulinemia, hipercolesterolemia e homocisteinemia. Daí a importância do primeiro passo, que é a identificação precoce dos comportamentos determinantes de risco, para posterior intervenção educativa com a finalidade de prevenção primordial e primária ${ }^{21}$. Com estes pressupostos, no segundo semestre de 1999, a Unidade de Epidemiologia Clínica do InCor -
HCFMUSP, dentro do seu projeto de pesquisa, ensino e prestação de serviços à comunidade, em parceria com a Divisão Regional de Ensino Centro-Oeste da Secretaria de Estado da Educação do Governo de São Paulo, desenvolveu o programa de "Práticas de Educação em Saúde e Epidemiologia Cardiovascular", com o objetivo de coletar, divulgar e discutir informações de saúde e prevenção de doenças cardiovasculares junto aos alunos de $5^{\mathrm{a}}$ a $8^{\mathrm{a}}$ séries do ensino fundamental. Estes alunos receberam orientação, fundamentada no seu risco pessoal avaliado por questionário. Tanto a coleta de informações como as palestras dialogadas foram realizadas por alunos do curso de graduação médica da Faculdade de Medicina da Universidade de São Paulo, como parte de suas atividades curriculares. De tal forma que as informações colhidas serviram como estudo piloto para o desenvolvimento do levantamento que agora se apresenta.

O objetivo do presente trabalho é estudar a prevalência do risco cardiovascular associado ao estilo de vida de escolares matriculados entre a $5^{\mathrm{a}}$ e a $8^{\mathrm{a}}$ séries do ensino fundamental, em função do sexo, série escolar e condição de escola pública ou privada. O reconhecimento precoce dos riscos passíveis de correção pode servir para fundamentar o desenvolvimento de ações preventivas na comunidade escolar.

\section{Métodos}

No início do projeto, em 2000, o levantamento oficial mostrou que 4335 escolas públicas e privadas do município de São Paulo estavam divididas em 13 distritos de ensino 22 . Optamos por estudar as Regionais de Ensino Centro e Centro-Oeste-Sul por estarem mais próximas da instituição coordenadora da pesquisa. $O$ conjunto de salas de aula dessas regionais representa $25 \%$ de todas as salas de aula no município de São Paulo. Estas regionais se diferenciam das demais por apresentarem número equivalente de instituições públicas e privadas, ao contrário das restantes onde o número de escolas públicas é quase sete vezes maior. Optamos pelas $5^{\mathrm{a}}, 6^{\mathrm{a}}, 7^{\mathrm{a}}$ e $8^{\mathrm{a}}$ séries por representarem um ciclo completo do sistema de ensino e corresponderem ao início da adolescência, período caracterizado por mudanças no plano individual e social que leva à experimentação de comportamentos adultos como o fumo e o consumo de bebidas alcoólicas. Do total de 4002 salas de aula existentes nas escolas das regiões escolhidas, foram selecionadas aleatoriamente 17| salas. Destas, durante os anos letivos de 2001 e 2002, foram visitadas 87 salas de 30 escolas que aceitaram participar do estudo, sendo I I das 502 escolas da Regional de Ensino Centro e 19 das 8 I 5 escolas da Regional de Ensino Centro-Oeste-Sul. Com relação aos bairros foram: dois da Consolação, três do Limão, quatro de Perdizes, um de Santa Cecília, um da Sé, três do Alto de Pinheiros, dois do Butantã, um do Campo Belo, um do Itaim-Bibi, quatro da Lapa, um de Moema, um do Morumbi, dois de Pinheiros, quatro do Rio Pequeno. Todos os alunos presentes no dia da visita, com exceção de 19 , foram incluídos na amostra, totalizando 2125 estudantes, sendo 47,3\% matriculados em escolas privadas e 52,7\% em escolas públicas; $50,5 \%$ do sexo feminino e $49,5 \%$ do masculino. A amostra final representa cerca de $2 \%$ do total de alunos matriculados nas regiões estudadas.

O desenho do estudo é do tipo transversal para levantamento das prevalências de sobrepeso, obesidade e algumas características do estilo de vida dessa população. Para a coleta de informações foi 
NoBRE MRC ET AL.

desenvolvido um questionário para ser preenchido pelo próprio aluno, pré-codificado, com perguntas fechadas, divididas em cinco blocos. 0 primeiro para coleta de dados sociodemográficos e os demais relacionados ao estilo de vida, com ênfase nos riscos cardiovasculares como inatividade física, uso de bebidas alcoólicas, tabagismo e hábitos alimentares. Sempre que existentes e adequados para a população em estudo, foram utilizados instrumentos pré-testados para compor o questioná$\mathrm{rio}^{23,24}$. Segundo normas do IBGE, foram consideradas cinco categorias para o escolar se classificar quanto à característica cor ou raça: branca, preta, amarela, parda e indígena. A aplicabilidade do questionário quanto ao tempo de preenchimento foi ajustada para uma média de 25 minutos em avaliação piloto. O pré-teste validou a compreensão do vocabulário e a clareza de significados. As questões que apresentaram maior percentual de resposta em branco foram reformuladas ou descartadas. Vinte e nove estudantes da área da saúde foram treinados para conduzir a aplicação do questionário de forma a responder eventuais dúvidas dos escolares com o cuidado de não induzir as respostas, recomendando que deixassem em branco as questões que não soubessem responder. Utilizando-se instrumentos e procedimentos recomendados para estudos de campo, foi medido o peso, a estatura, e calculado o índice de massa corpórea.

Como padrão validado, foi utilizado o questionário norte-americano para avaliação de risco em adolescentes ${ }^{23}$, conhecido como YHBSS-CDC, do qual foram retiradas e adaptadas as questões referentes ao tabagismo, ao consumo de álcool, a questão do consumo de frutas e verduras. Com relação à quantidade de atividade física realizada pelo escolar, foi solicitado que assinalasse uma alternativa correspondente à sua situação entre as quatro seguintes: ando muito pouco, passo a maior parte do dia sentado; me movimento bastante, mas não subo escadas e não carrego peso; subo escadas e carrego objetos leves; carrego objetos pesados, ou faço outros exercícios físicos intensos ${ }^{25}$. Foram classificados como sedentários os que referiram andar muito pouco e passar a maior parte do dia sentado. Os riscos com relação ao tabagismo e ao uso de bebidas alcoólicas foram atribuídos aos escolares que experimentaram o cigarro e o álcool, mesmo que 0 uso atual não tenha sido considerado regular ${ }^{26}$. 0 conjunto de alunos com sobrepeso ou obesidade foi definido a partir do percentil 85 do índice de massa corpórea, tendo como referência o estudo NHANES I ${ }^{27}$. Com relação aos hábitos alimentares, foi indagado quantas vezes no dia anterior o escolar havia se alimentado dos diferentes grupos alimentares, entre as seguintes alternativas: nenhuma, uma, duas, três, quatro, cinco ou mais vezes. Os grupos alimentares foram representados por laticínios como fonte de cálcio; legumes crus, legumes cozidos, frutas e sucos naturais como alimentos reguladores; salgadinhos fritos e industrializados como junk food; embutidos e enlatados como alimentos com alto teor de sódio; manteiga, maionese e fritura como alto teor de gordura saturada; adição de sal ao alimento já preparado; e refrigerantes.

As prevalências estão apresentadas de forma gráfica quanto ao sexo, série cursada e tipo de escola. Foi realizado teste de tendência linear para proporções nas séries escolares, via modelos lineares generalizados, linkidentidade e família binomial28. O teste Qui-quadrado para tabelas foi utilizado para se testar as diferenças de prevalência dos fatores de risco entre tipos de escola, pública e privada.
Utilizou-se a análise de correspondência múltipla ${ }^{29}$ para a análise de perfil de resposta das variáveis de consumo alimentar e tipificação dos indivíduos em perfis de consumo. A consolidação dos perfis foi feita por meio da análise de agrupamentos ${ }^{30}$.

Para os testes e modelos estatísticos utilizou-se o software Stata 8.0 (Stata Corp, 2003) e para análise de correspondência e agrupamentos o SPAD 3.5 (CISIA-CERESTA, 1995).

\section{Resultados}

A análise de correspondência múltipla é uma técnica exploratória multivariada que, diferentemente dos modelos clássicos, faz o cruzamento multifatorial sem condicionar previamente a relação entre as variáveis como dependentes e independentes, e tem como objetivo a representação gráfica de um grande conjunto de variáveis categóricas por meio da redução do espaço original de representação destas. Por meio da contribuição dos eixos na variabilidade total, identifica-se associações plausíveis de interpretaçãa. Sob as coordenadas destes eixos aplica-se a análise de agrupamento, ou cluster analysis, que busca por meio de técnicas adequadas a consolidação e classificação dos indivíduos. A utilização desta técnica permite que se encontre agrupamentos de indivíduos que compartilham perfil semelhante de resposta nas variáveis estudadas. Uma vez interpretados os eixos e associações, estes agrupamentos também são interpretáveis de acordo com suas características principais. Utilizou-se a técnica para se encontrar agrupamentos de indivíduos quanto ao perfil de hábito alimentar, de acordo com os seguintes tipos de alimentos: laticínios; legumes crus, legumes cozidos, frutas e sucos naturais; salgadinhos fritos e industrializados; embutidos e enlatados; manteiga, maionese e fritura; adição de sal ao alimento já preparado; e refrigerantes.

Identificou-se de forma clara, pela análise de agrupamento a presença de dois perfis distintos de hábito. No canto superior esquerdo da Figura I agrupa-se um conjunto de respostas caracterizadas pela colocação adicional de sal à comida já preparada, menor consumo no dia anterior dos grupos alimentares representados pelas frutas e laticínios, por um lado, e maior consumo dos grupos alimentares representados pelos refrigerantes, gorduras e salgadinhos, de outro lado. Aos indivíduos deste agrupamento foi atribuída a condição de hábito alimentar inadequado.

A média de idade foi de $12,96 \pm 1,31$ e a mediana 13. Quanto à cor ou raça: $65 \%$ se declararam brancos, $20 \%$ pardos, $8 \%$ pretos, $4,4 \%$ amarelos e 2,6\% indígenas. Os brancos e amarelos predominaram nas escolas privadas; pretos, pardos e indígenas nas públicas.

O consumo de bebidas alcoólicas pelo menor uma vez na vida foi de $62,6 \%$, em proporção maior nas escolas privadas comparado às públicas, tanto no sexo feminino ( $68,4 \%$ contra $58,9 \%$, com $p=0,00$ I) como no masculino $(66,8 \%$ contra $56,6 \%$, com $p=0,002)$. O consumo de cigarro pelo menor uma vez na vida de $23,1 \%$, não se diferenciou com relação à escola, embora com tendência a ser maior nas meninas das escolas públicas ( $25,2 \%$ contra $24,5 \%$, com $p=0,805$ ) e nos meninos das escolas privadas ( $22,8 \%$ contra $19,4 \%$, com $p=0,2 \mid 2)$. O uso regular, com frequêencia de algumas vezes no mês a todos os dias, foi de $9,2 \%$ para o álcool e 4,9\% para o fumo, no total dos alunos estudados. $\mathrm{Na} 8$ a série, 3,7\% referiram fumar todos os dias 
Figura I - Agrupamentos quanto ao perfil de freqüência e hábito alimentares definidos pelas análises de agrupamento e correspondência

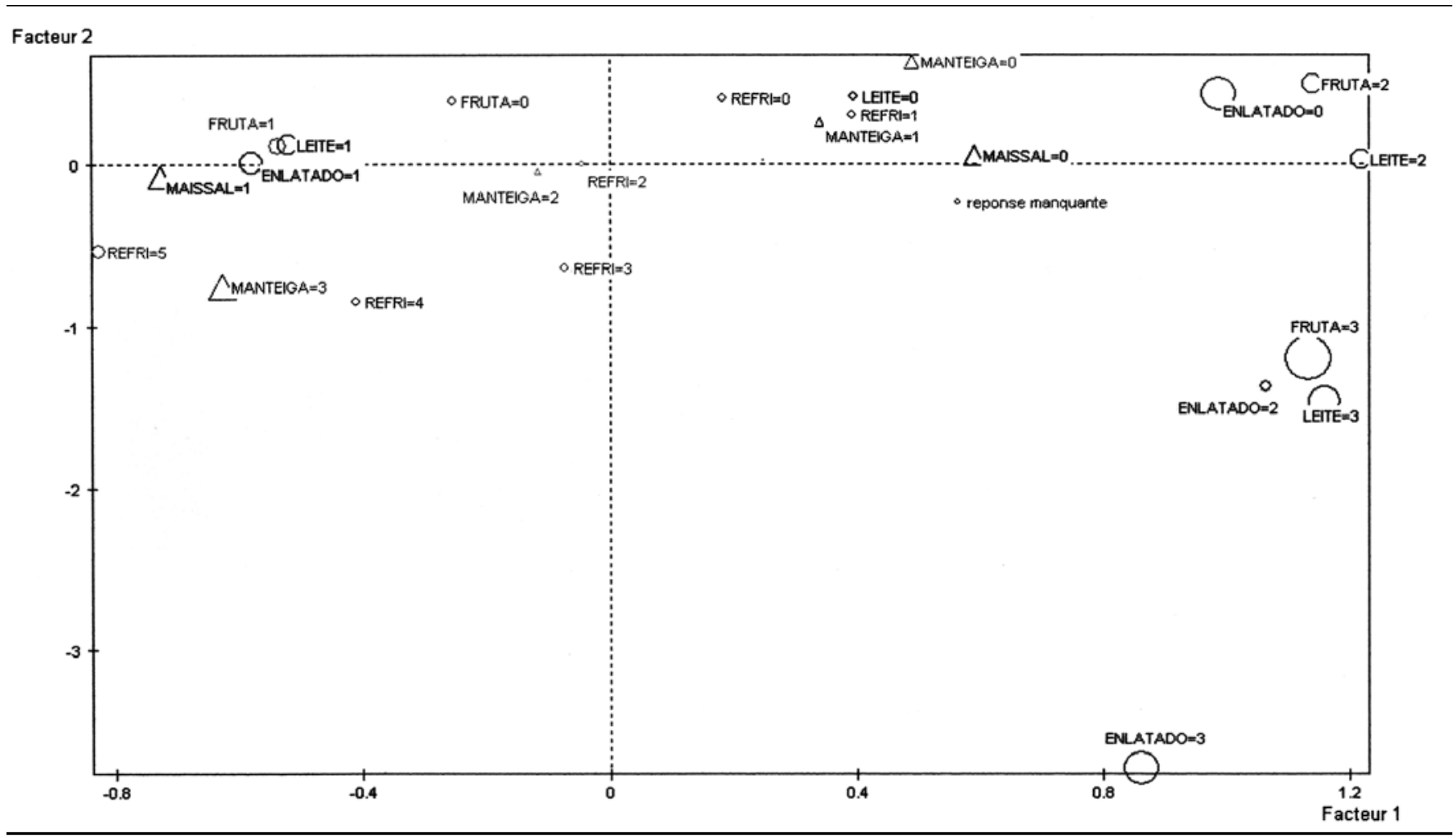

Maissal: hábito de adicionar sal ao alimento já preparado: $0=$ não I = sim

Refri(gerante), fruta, leite, manteiga e enlatado; em número de vezes que foram consumidos no dia anterior: 0, I, 2, 3, 4, 5 ou mais vezes

e 3,1\% semanalmente, enquanto 5,7\% relataram consumir bebida alcoólica diária ou semanalmente. Entre os mais velhos, o uso mais freqüente da bebida, algumas vezes na semana ou todos os dias, se deu em maior proporção na escola pública, referida por 35 alunos, comparado com os 15 alunos de escolas privadas, invertendo a observação inicial de consumo alguma vez na vida.

O sedentarismo observado em 15,4\% dos alunos, foi mais prevalente nas escolas públicas que nas privadas, tanto no sexo feminino (I8,2\% contra I2,3\%, com $p=0,0 \mid 0)$, como no masculino (I6,8\% contra $12,2 \%$, com $p=0,042$ ). Este último achado é confirmado pela ausência nas aulas de educação física, maior nas escolas públicas, mais uma vez observada tanto no sexo feminino ( $11,8 \%$ contra $5,7 \%$, com $p=0,00 I)$, como no masculino (7, I \% contra 2,4\%, com $p=0,00 I)$. As adolescentes do sexo feminino freqüentam menos as aulas de educação física que os do sexo masculino (8,9\% contra 4,9\%, com p = 0,0002). O sedentarismo é maior na escola pública, em ambos os sexos. No sexo feminino, ele não se altera em função da série. Nos adolescentes do sexo masculino que freqüentam escola privada, o sedentarismo foi menor nos mais velhos, em contraposição com os que freqüentam a escola pública, onde ele aumenta com a idade.

A obesidade não se diferenciou entre as escolas públicas e privadas, embora com tendência a ser mais prevalente nas privadas $(6,7 \%$ contra $5,6 \%$, com $p=0,486)$ e no sexo feminino ( $11,3 \%$ contra $8,1 \%$, com $p=0,094)$. No total a prevalência do sobrepeso e obesidade foi de
$24 \%$, sendo $26 \%$ nas escolas privadas e $22 \%$ nas públicas ( $p=0,035$ ). O hábito alimentar inadequado predominou em ambos os sexos nas privadas, sendo encontrado em 53,3\% do total da população estudada.

$\mathrm{Na}$ Figura 2 observa-se que com o progredir das séries cresce 0 risco do uso de cigarro e bebida alcoólica. Entre a $5^{\mathrm{a}}$ e a $8^{\mathrm{a}}$ série, dobra o uso de bebidas alcoólicas, em ambos os sexos. Triplica a proporção de adolescentes do sexo masculino e quintuplica a proporção no sexo feminino que experimentam o cigarro. A tendência crescente de experimentação da bebida alcoólica e do cigarro com o progredir das séries escolares se observa tanto nas escolas públicas como nas privadas. Em contraposição, reduz o risco de hábito alimentar inadequado e excesso de peso, independentemente do sexo e da condição pública ou privada da escola. A prevalência percentual do sobrepeso cai de 17,0 para 13,8 da 5a para a $8^{a}$ série, e a obesidade cai de 9, I para 7, I no total da população estudada.

\section{Discussão}

Os fatores biológicos, que conferem risco para o desenvolvimento de doenças crônicas, estão diretamente relacionados com as opções de comportamento feitas na infância e adolescência. Resultados do levantamento norte-americano YRBSS de 200 I demonstraram que a maioria dos comportamentos de risco associados ao desenvolvimento de câncer e aterosclerose se iniciam nessa faixa etária ${ }^{31}$, com a aquisição de hábitos como o tabagismo, desvios alimentares, sedentarismo, uso de álcool e 


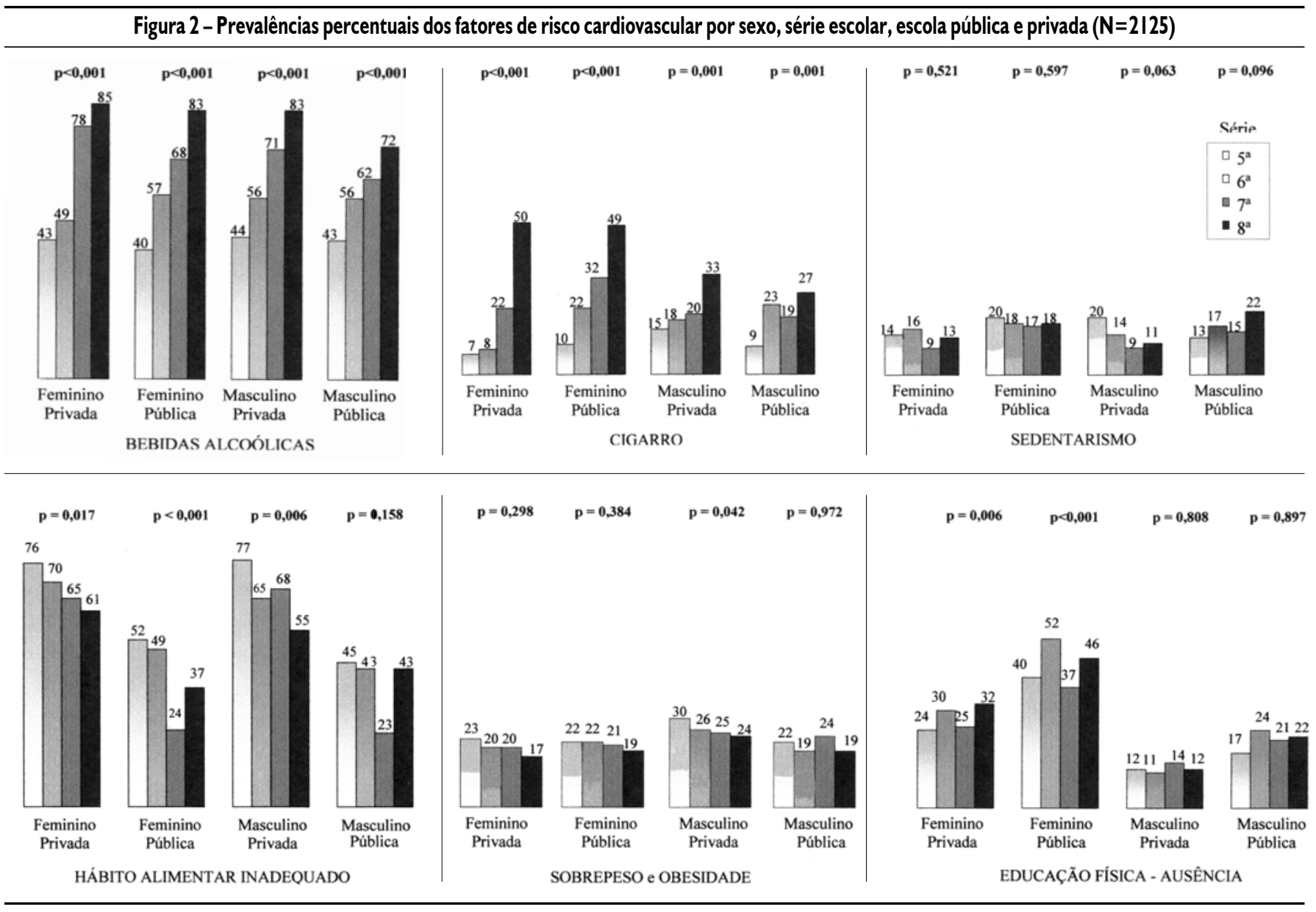

outras drogas. Estes comportamentos propiciam o aparecimento ou agravam diferentes condições associadas à saúde como o estresse, hipertensão arterial, obesidade, hiperinsulinemia, hipercolesterolemia e homocisteinemia. Todos considerados fatores biológicos de risco passíveis de reversão. Daí a importância da identificação precoce destes comportamentos determinantes de risco para que sua prevenção possa ser obtida por meio de processos educativos ${ }^{32}$. As estratégias básicas para mudança dos determinantes sociais que conferem comportamentos de risco devem priorizar a comunidade, a família e a escola para o desenvolvimento de programas de prevenção primordial ${ }^{33}$.

Entre os 12 e 17 anos, não comeram pelo menos cinco porções de frutas e vegetais por dia, nos sete dias que antecederam a pesquisa, $78,6 \%$ dos norte-americanos ${ }^{31}$, percentual semelhante àquele por nós observado na $5^{\mathrm{a}}$ série como hábito alimentar inadequado. Acima do percentil 85 de referência de peso se apresentavam 24\% dos norteamericanos, a mesma proporção por nós observada.

No levantamento HBSC realizado entre 1989 e 1994, no Canadá e países da Europa, foi universal o aumento acentuado do número de adolescentes que experimentam o cigarro entre os II e os 15 anos $^{34}$. Aos $\mid$ anos, $75 \%$ dos meninos canadenses e $70 \%$ das meninas experimentaram o álcool. Aos 15 anos, este percentual sobe para $94 \%$ em ambos os sexos. Diminui o número de canadenses interessados em práticas esportivas, principalmente no sexo feminino. Embora o nosso levantamento tenha mostrado prevalências menores, as magnitudes de elevação dos riscos foram bastante semelhantes, inclusive a redução de interesse pela prática de atividade física nas meninas.

A Organização Mundial da Saúde divulgou, em 2000, o seu quarto levantamento HSBC sobre o comportamento relacionado à saúde de I 20 mil adolescentes entre II e I5 anos de idade de 28 países $^{35}$. A maior porcentagem de adolescentes que fumavam todos os dias foi encontrada na Groenlândia 56\% das meninas e 45\% dos meninos de I 5 anos. Metade dos adolescentes na mesma idade bebia pelo menos uma vez por semana no País de Gales. Na Inglaterra 24\% das meninas e $21 \%$ dos meninos fumavam todos os dias, e $36 \%$ das meninas e $47 \%$ dos meninos bebiam todas as semanas. Entre os norte-americanos de 15 anos, $13 \%$ das meninas e 12\% dos meninos fumavam diariamente, enquanto $15 \%$ das meninas e $23 \%$ dos meninos consumiam álcool todas as semanas. As prevalências por nós observadas na oitava série foram menores do que as menores prevalências internacionais relatadas, cerca de metade para o cigarro e um terço para a bebida, comparadas às norte-americanas.

As prevalências por nós relatadas estão mais próximas de outras experiências brasileiras ${ }^{36,37,38,39}$, com a ressalva que o limite superior da faixa etária no nosso trabalho apresenta diferença a menor de três anos. 
As nossas observações quanto ao tabagismo concordam com a afirmação anterior de que o uso no Brasil é menor do que nos EUA ${ }^{36}$, embora não possamos descartar a possibilidade de referência subestimada por parte dos escolares. Em estudo de dimensão nacional, não foi verificada diferença importante na prevalência do consumo de bebidas, cigarro e outras drogas entre as 10 maiores capitais brasileiras. Como também não foi observada diferença importante entre o levantamento realizado em 1987 e repetido em 1997 nas mesmas localidades ${ }^{36}$. O levantamento de cerca de 15.000 estudantes brasileiros, entre 10 e 18 anos, mostrou que o uso durante a vida de álcool foi de $78 \%$, e do cigarro $31,2 \%$, sendo $62,6 \%$ e 23, $1 \%$ os percentuais por nós observados. No levantamento nacional, o uso freqüente, definido como em seis ou mais dos últimos 30 dias, foi de I5\% para a bebida alcoólica e 6,2\% para o cigarro, enquanto o nosso levantamento mostrou ser de 9,2\% e 4,9\%, respectivamente. A diferença poderia ser explicada pelo incremento crescente desses hábitos entre os 15 e os 18 anos, idades que conferem o limite superior das populações que estão sendo comparadas.

O estudo de 1.187 moradores de Pelotas ${ }^{37}$, entre 10 e 19 anos de idade, mostrou prevalência de 26,7\% para o "fumo alguma vez na vida", incluindo fumo atual, fumo ocasional e ex-fumante, comparado aos $23,1 \%$ por nós observados entre os I I e os 15 anos. No estudo de Pelotas, o tabagismo atual, definido como fumar um ou mais cigarros nos últimos 30 dias, apresentou prevalência de $1,2 \%$ entre os 10 e os 13 anos, $10,9 \%$ entre os 14 e os 16 anos e $25,8 \%$ entre os 17 e os 19 anos, caracterizando a magnitude crescente do envolvimento com 0 cigarro.

O nosso achado de 62,6\% para consumo de bebidas alcoólicas e de $23,1 \%$ para o uso de cigarro pode ser comparado com outro levantamento, de $|.44|$ alunos do ensino médio e fundamental de Brasília, que encontrou 67,2\% para o consumo alguma vez na vida de bebidas alcoólicas e $28,7 \%$ para o fumo ${ }^{38}$. Estudando escolares mais velhos de Ribeirão Preto ${ }^{39}$, incluindo a 8 a série do ensino fundamental e as três séries do ensino médio, o uso alguma vez na vida de álcool foi de $88,9 \%$ e do cigarro $37,7 \%$, sugerindo que a escalada do consumo dessas substâncias se mantém crescente durante o ensino médio.

As prevalências observadas quanta a condição de sobrepeso e obesidade neste estudo estão de acordo com inquérito domiciliar representativo da população de adolescentes brasileiros, que é menor do que a esperada, tomando como referência os adolescentes dos países mais industrializados ${ }^{18}$. A maior proporção de sobrepeso e obesidade entre adolescentes de escolas privadas, comparadas à de escolas públicas, também foi observado em escolas da cidade do Rio de Janeiro ${ }^{40}$.

\section{Conclusão}

O crescimento marcante do uso de bebidas alcoólicas e cigarro, entre a $5^{\mathrm{a}}$ e a $8^{\mathrm{a}}$ série, acentua a importância de intervenções preventivas na adolescência, sugerindo que, quanto mais cedo se iniciarem as intervenções preventivas, melhores poderiam ser os resultados. Os nossos achados também recomendam práticas de educação em saúde que objetivem implementar hábitos alimentares saudáveis e a quantidade de atividade física, com especial atenção para o sexo feminino. 0 reconhecimento precoce dos riscos passíveis de correção deverá servir para fundamentar o desenvolvimento de ações preventivas na comunidade escolar.

\section{Agradecimentos}

Os autores agradecem às escolas participantes do projeto e as colaborações de Marcio Polydoro, dos alunos do curso de graduação da Faculdade de Medicina da USP que cursaram a disciplina "Práticas de Educação em Saúde e Epidemiologia Cardiovascular", como também, dos alunos que fizeram a iniciação científica na Unidade de Epidemiologia Clínica do InCor - HCFMUSP pela dedicação demonstrada nas atividades com os alunos das escolas de ensino fundamental.

Conflito de interesse: não há.

\section{SUMMARY \\ Prevalence of overweight, obesity and lyfe style ASSOCIATED WITH CARDIOVASCULAR RISK AMONG MIDDLE SCHOOL STUDENTS}

BACKGROUND. To study the prevalence of cardiovascular riskassociated to the lifestyle of school children from the $5^{\text {th }}$ to $8^{\text {th }}$ grade, in public and private schools.

METHODS. Eighty seven randomly selected school rooms were visited adding up to 2, 125 students who completed the questionnaire and whose body mass index was calculated. This sample represents around $2 \%$ of students from two school districts in the city of Sao Paulo.

RESULTS. Of the 2, 125 students, 24\% were overweight or obese, $53.3 \%$ presented inappropriate food habits, $15.4 \%$ were sedentary, $62.6 \%$ drank alcohol, and $23.1 \%$ smoked. Between the $5^{\text {th }}$ and $8^{\text {th }}$ grade, the number of students who drank alcohol doubled and the number of male and female adolescents who tried out smoking increased 3-fold and 5-fold, respectively. Conversely, inappropriate food habits decreased. This situation is found in $40 \%$ of students from public schools and in $58 \%$ of those in private schools, by the time they reach the $8^{\text {th }}$ grade. Overweight and obesity are more prevalent in private schools and the reduction noted as grades progress did not reach a significance level. Sedentarism is more prevalent in public schools. In private ones, sedentarism is lower among older students, contrary to public schools, where it increases with age. Female adolescents attend fewer physical education classes. Inappropriate food habits were characterized by the habits of adding more salt to pre-prepared food, lowerintake of dairy products, fruitand vegetables also a higher intake of soft drinks, butter and snacks.

CONCLUSION. Early detection of these cardiovascular risks associated to the lifestyle of these school children endorses the preventive practice of providing health education in schools. [Rev Assoc Med Bras 2006; 52(2): I 18-24]

KeY Words: Epidemiology. Cardiovascular diseases. Lifestyle. Adolescent. Primary schools. Health education.

\section{REFERÊNCIAS}

I. Lloyd-Jones DM, Larson MG, BeiserA, Levy D. Lifetime risk of developing coronary heart disease. Lancet 1999;353:89-92.

2. World Health Organization. The World Health Report 1999. Statistical Annex. [cited 20 apr 2000].Avaliable from: http://www.who.int/. 
NOBRE MRC ET AL.

3. Governo do Estado de São Paulo. Fundação Sistema Estadual de Análise de Dados. Seade. Disponível em: http://www.seade.gov.br/. [citado 20 abr 2000].

4. Prefeitura do Município de São Paulo. Secretaria de Saúde. Programa de Aprimoramento das informações de mortalidade - PROAIM. Disponível em: http://www.prodam.sp.gov.br/. [citado 20 abr 2000].

5. Francoso LA, Coates V. Anatomicopathological evidence of the beginning of atherosclerosis in infancy and adolescence. Arq Bras Cardiol 2002;78: $131-42$

6. Newman WP 3rd, Freedman DS, Voors AW, Gard PD, Srinivasan SR, Cresanta JL, et al. Relation of serum lipoprotein levels and systolic blood pressure to early atherosclerosis. The Bogalusa Heart Study. N Engl J Med 1986; 3 |4: | 38-44.

7. Pan-American Health Organization. C.A.R.M.E.N. - Conjunto de Acciones para la Reducción Multifactorial de las Enfermedades NoTansmisibles. What are NCD risk factor Available from: http:// www. paho.org/english/ad/dpc/nc/carmen -info.htm. [cited 25 feb 2004].

8. Kannel WB, Dawber TR, Kagan A, Revotskie N, Stokes J 3rd. Factors of risk in the development of coronary heart disease-six year follow-up experience. The Framingham Study. Ann Intern Med 1961;55:33-50.

9. Keys A, editor. Seven countries: a multivariate analysis of death and coronary heart disease. Cambridge: Harvard University Press; 1980.

10. Intersalt Cooperative Research Group: an international study of electrolyte excretion and blood pressure. Results for 24 hour urinary sodium and potassium excretion. BMJ | 988; 297(6644):319-28.

I I. Kannel WB, McGee DL, Castelli, WP. Latest perspectives on cigarette smoking and cardiovascular disease: the Framingham Study. J Card Rehabil 1984: 4:267-77.

12. Leon AS, Connett J, Jacobs DR Jr, Rauramaa R. Leisure-time physical activity levels and risk of coronary heart disease and death. The Multiple Risk Factor Intervention Trial. JAMA 1987; 258:2388-95.

13. Powell KE, Thompson PD, Caspersen C], Kendrick JS. Physical activity and the incidence of coronary heart disease. Annu Rev Public Health 1987;8:253-87.

14. Criqui $\mathrm{MH}$. The roles of alcohol in the epidemiology of cardiovascular diseases. Acta Med Scand Suppl 1987;717:73-85.

15. Regan TJ. Alcohol and the cardiovascular system. JAMA 1990; 264:37781.

16. Popkin, BM. The nutrition transition in low-income countries: an emerging crisis. Nutr Rev 1994;52:285-98.

17. Sichieri R, Recine E, Everhart JE. Growth and body mass index of Brazilians ages 9 through 17 years. Obes Res 1995;3(Suppl 2): I 17s-2 Is.

18. Neutzling MB, Taddei JA, Rodrigues EM, Sigulem DM. Overweight and obesity in Brazilian adolescents. Int J Obes Relat Metab Disord 2000;24:869-74.

19. Webber LS, Osganian SK, Feldman HA, Wu M, McKenzie TL, Nichaman $\mathrm{M}$, et al. Cardiovascular risk factors among children after a 2 I/2-year intervention-The CATCH Study. Prev Med 1996;25:432-4I.

20. Beilin L, Burke V, Milligan R. Strategies for prevention of adult hypertension and cardiovascular risk behaviour in childhood. An Australian perspective. J Hum Hypertens 1996; I O(Suppl I):S5 I-4.

21 . Washington RL. Interventions to reduce cardiovascular risk factors in children and adolescents. Am Fam Physician 1999;59:221 I-8.

22. Cadastro de escolas regulares e unidocentes da matrícula inicial. Redes de ensino: estadual, municipal e particular. Ano base 1999. Censo MEC. Disponível em: http://www.educacao.sp.gov.br/pesquisa/ download arquivo escolas. [citado 10 jul 2000].

23. Centers for Disease Control and Prevention. Youth risk behavior surveillance system. 1999 YRBSS information and results. Avaliable from: http://www.cdc.gov/nccdphp/ dash/yrbs. [cited I 0 jun 2000].
24. BRASIL. Instituto Brasileiro de Geografia e Estatística. Censo Demográfico 2000. Questionário Básico CD I.0I IBGE. Pesquisa Nacional por Amostra de Domicílios. Disponível em: http://wwwl.ibge.gov.br/ibge/ estatistica/populacao/trabalhoerendimento/pnad99/ metodologia99.shtm. [citado 25 jul 2000].

25. Organização Mundial da Saúde. Organização Pan-Americana da Saúde Programa de Intervenção Integrada de Alcance Nacional em Doenças Não-Transmissíveis (CARMEN/CINDI) - Protocolo e Diretrizes. Brasília; 1997

26. Sherratt E, MacArthur C, Cheng KK. West midlands young people's lifestyle survey 1995-1996. London: Final Report. University of Birmingham; 1996.

27. Must A, Dallal GE, Dietz WH. Reference data for obesity: 85th and 95th percentiles of body mass index (wt/ht2) and triceps skinfold thickness. Am J Clin Nutr 1991;53:839-46.

28. McGullagh P, Nelder JA. Generalized Linear Models. New York: Chapman and Hall; 1989.

29. Lebart M, Morineau A, Piron M. Statistique exploratoire multodomensionelle. Paris: Dunod; 1995.

30. Pereira JC. Análise de dados qualitativos: estratégias metodológicas para ciências da saúde, humanas e sociais. São Paulo: EDUSP; 1999.

31. Grunbaum JA, Kann L, Kinchen SA, Williams B, Ross JG, Lowry R, et al. Youth risk behavior surveillance-United States, 2001. J Sch Health 2002;72:313-28.

32. Washington RL. Identifying at-risk children and adolescents is the first step in modifying or preventing these risk factors. Interventions to reduce cardiovascular risk factors in children and adolescents. Am Fam Physician 1999;59(8):221 I-8

33. De Backer GG, Maes L, Van de Mieroop E. Issues for cardiovascular disease risk factor development in Europe. Prev Med 1999;29(6 Pt 2):S96-101.

34. Health Canada. Comprehensive School Health. Health behaviours in school aged children survey. Available from: http://www.hbsc.gc.ca/main/ hc/web/datahpsb/children/ english/sec4-I.htm, . [cited 23 jul 2004].

35. Ciment J. WHO study examines teenage health in 28 countries. BMJ 2000;320(7232):402.

36. Galduroz JC, Noto AR, Nappo SA, Carlini EA. Trends in drug use among students in Brazil: analysis of four surveys in 1987, 1989, 1993 and 1997. Braz J Med Biol Res 2004;37:523-3I.

37. Malcon MC, Menezes AM, Chatkin M. Prevalence and risk factors for smoking among adolescents. Rev Saude Publica 2003;37: I -7.

38. Godoi AM, Muza GM, Costa MP, Gama ML. Use of psychoactive substances among private school children. Rev Saude Publica. 1991 Apr;25(2): I50-6.

39. Muza GM, Bettiol H, Muccillo G, Barbieri MA. The consumption of psychoactive substances by adolescents in schools in Ribeirao Preto, SP (Brazil). I-Prevalence of consumption by sex, age and substance. Rev Saude Publica 1997;31:21-9

40. Gross R, De Lima FD, De Freitas C], Gross U. The relationships between selected anthropometric and socio-economic data in schoolchildren from different social strata in Rio de Janeiro, Brazil. Rev Saude Publica 1990 Feb;24(I): | I-9.

Artigo recebido: 14/09/04

Aceito para publicação: 22/01/06 